\title{
Lorenz Kaotik Sisteminin Doğrusal Geri Beslemeli, Yüksek Kazanç, Yüksek Frekans ve Model Öngörülü Kontrol ile Denetlenmesi
}

*1 Murat Erhan Çimen, ${ }^{2}$ Muhammed Ali Pala, ${ }^{3}$ Ömer Faruk Boyraz, ${ }^{4}$ Zeynep Garip, ${ }^{5}$ Akif Akgül, ${ }^{6}$ Mustafa Zahid Yıldız, ${ }^{7}$ Ali Fuat Boz,

1*Sakarya Uygulamalı Bilimler Üniversitesi, Elektrik ve Elekt. Müh. Bölümü, Sakarya, Türkiye,muratcimen@subu.edu.tr, ${ }^{2}$ Sakarya Uygulamalı Bilimler Üniversitesi, Elektrik ve Elekt. Müh. Bölümü, Sakarya, Türkiye,pala@ subu.edu.tr,

${ }^{3}$ Anadolu Isizu Otomativ Sanayi ve Ticaret A.Ş., Ar-Ge Merkezi, Kocaeli, Turkiye, omer.boyraz@isuzu.edu.tr, ${ }^{4}$ Sakarya Uygulamalı Bilimler Üniversitesi, Bilgisayar Müh. Bölümü, Sakarya, Türkiye, zbatik@ subu.edu.tr,

${ }^{5}$ Hitit Üniversitesi, Bilgisayar Mühendisliği Bölümü, Çorum, Türkiye, akifakgul@ hitit.edu.tr,

${ }^{6}$ Sakarya Uygulamalı Bilimler Üniversitesi, Elektrik ve Elekt. Müh. Bölümü, Sakarya, Türkiye,mustafayildiz@ subu.edu.tr,

${ }^{7}$ Sakarya Uygulamalı Bilimler Üniversitesi, Elektrik ve Elekt. Müh. Bölümü, Sakarya, Türkiye,afboz@ subu.edu.tr,

Araştırma Makalesi

Geliş Tarihi: 05.10.2020

Kabul Tarihi: 14.03.2021

$\ddot{O} z$

Günümüze kadar gelişen ve geliştirilmeye devam edilen, doğrusal veya doğrusal olmayan, zamanla değişen veya zamanla değişmeyen sistemler için birçok kontrol yöntemleri bulunmaktadır. Bu çalışmada doğrusal olmayan Lorenz Kaotik sisteminin kontrolünde daha önceden bu sisteme uygulanmamış olan integratör içeren model öngörülü kontrol yöntemi uygulamıştır. Bu yöntemin yanı sıra aynı sisteme farklı doğrusal olmayan kontrol yöntemleri de uygulanarak Lorenz Kaotik sisteminin kontrolü gerçekleştirilmiştir. Seçilen kontrol yöntemlerinde geri beslemeli kontrol, yüksek kazanç kontrol, yüksek frekans kontrol ve model öngörülü kontrol teknikleri kullanılmıştır. Ayrıca kullanılan yöntemler matematiksel olarak elde edilmiş, avantaj ve dezavantajlarını ortaya konulmuştur. Ayrıca literatürde Lorenz sistemi için üretilmiş olan kontrol kuralları kullanılarak performans açısından karşılaştırmalar yapılmıştır. Sonuçta doğrusal olmayan bu tip kontrol yöntemlerinin Lorenz kaotik sisteminin kontrol edebildiği gösterilmiş. Ardından avantaj ve dezavantajları sonuçlar bölümünde tartışılmış ve ileriye yönelik çalışmalar hakkında bilgiler verilmiştir.

Anahtar Kelimeler: Kaotik sistem, geri beslemeli kontrol, yüksek kazanç kontrolü, yüksek frekans kontrolü, model öngörülü kontrol

\section{Control of Lorenz Chaotic System with Linear Feedback, High Gain, High Frequency and Model Predictive Control}

${ }^{* 1}$ Murat Erhan Çimen, ${ }^{2}$ Muhammed Ali Pala, ${ }^{3}$ Ömer Faruk Boyraz, ${ }^{4}$ Zeynep Garip, ${ }^{5}$ Akif Akgül, ${ }^{6}$ Mustafa Zahid Yıldız, ${ }^{7}$ Ali Fuat Boz,

1*Sakarya University of Applied Sciences, Electrical and Electronic Eng. Dept., Sakarya Turkey,muratcimen@ subu.edu.tr, ${ }^{2}$ Sakarya University of Applied Sciences, Electrical and Electronic Eng. Dept., Sakarya Turkey,pala@ subu.edu.tr

${ }^{3}$ Anadolu Isizu Otomativ Sanayi ve Ticaret A.Ş., R\&D Merkezi, Kocaeli, Turkiye, omer.boyraz@isuzu.edu.tr ${ }^{4}$ Sakarya University of Applied Sciences, Computer Eng. Dept., Sakarya Turkey, zbatik@subu.edu.tr ${ }^{5}$ Hitit University, Computer Eng. Dept., Çorum, Turkey, akifakgul@ hitit.edu.tr

${ }^{6}$ Sakarya University of Applied Sciences, Electrical and Electronic Eng. Dept., Sakarya Turkey, mustafayildiz@ subu.edu.tr

${ }^{7}$ Sakarya University of Applied Sciences, Electrical and Electronic Eng. Dept., Sakarya Turkey, afboz@ subu.edu.tr

\begin{abstract}
There are many control methods for systems that have been developed and continued to be developed, linear or non-linear, timevarying or invariant. In this study, in the control of the nonlinear Lorenz Chaotic system, model predictive control method with integrator action, which has not been applied to this system before, is applied. In addition to this method, different nonlinear
\end{abstract}

*Sorumlu Yazar: Sakarya Uygulamalı Bilimler Üniversitesi, Elektrik ve Elektronik Müh. Bölümü, Sakarya Türkiye,muratcimen@subu.edu.tr, +90 2646160946 
control methods were applied to the same system and the control of the Lorenz Chaotic system was realized. Feedback control, high gain control, high frequency control and model predictive control techniques are used in the selected control methods. In addition, the methods used were obtained mathematically and their advantages and disadvantages were revealed. In addition, the control laws produced for the Lorenz system in the literature were used and comparisons were made in terms of performance. As a result, it has been shown that this type of nonlinear control methods can control the Lorenz chaotic system, and then the advantages and disadvantages are discussed in the results section and information about future studies is given.

Keywords: Chaotic system, feedback control, high gain control, high frequency control, model predictive control

\section{GİRIŞ}

Kaos doğada birçok yerde karşılaşıldığ 1 için direk olarak birçok mühendislik uygulamasında da görülmektedir. Kaotik bir sistemin temel bir özelliğgi, başlangıç koşullarına aşırı duyarlılığıdır; yani, başlangıç durumundaki küçük farklılıklar sistem durumunda olağanüstü farklılıklara yol açabilir. Kaotik sistemler; meteoroloji [1, 2], tıp [3, 4] çevre $[2,5]$, sanat $[6,7]$, kriptoloji $[8,9,10]$, haberleşme $[11,12]$, görüntü işleme [13], sinyal işleme [14, 15], yapay sinir ağları $[15,16]$ ve kontrol [17-26] alanlarında birçok çalışma yapılmış ve yapılmaya devam edilmektedir. Gerçek hayatta ise direk olarak karşılaşılan uygulamalara örnek olarak hava olaylarının tahmini, epilepsi hastalığının teşhisi, kanser hücrelerinin veya dokularının kaotik davranışlılarının incelenmesi, müzik notlarının veya senfonilerin üretilmesi, verilerin şifrelenmesi ve tekrar çözülmesi, kaotik karıştırıcı uygulamaları, motorların kaotik hılarının senkronizasyonlarında, kaotik davranış sergileyen elektrik makinelerinde vb. örnek olarak verilebilir. Kaotik olaylar kullanım alanına göre istenilen çıktıları üretmekte fakat bazı uygulamalarda bu çıktılar istenmemektedir. Kontrol açısından incelendiğinde ise kaotik davranışlar genellikle bastırılmak istenmektedir veya takip edilmek istenmektedir.

Literatürde kaos kontrolü, 1990'lı yılların başından beri geniş bir ilgi alanına sahiptir ve literatüde en popüler vakalardan birisi Lorenz kaotik sisteminin kontrol edilmesidir. Bu amaçla 1990 yılında Ott ve ark. kaotik bir çekicinin, mevcut bir sistem parametresinin sadece küçük zamana bağlı bozulmaları yaparak, çok sayıda olası zaman periyodik hareketinden herhangi birine dönüştürülebileceğini göstermiştir [18]. Vincent ve ark. Lorenz kaotik sistemi için bir strateji olarak doğrusal geri beslemeli kontrolü önermişlerdir. $\mathrm{Bu}$ tasarımda kararsız Tasarım geri besleme kontrolörü sistemi kontrolsüz olan Lorenz sisteminin denge noktalarından birine taşınmasına yönelik bir çalışma gerçekleştirmişleridir [19]. Yeap ve Ahmed, Lorenz sistemini kontrol etmek için çok katmanlı ileri beslemeli bir sinir ağı kullanarak geri besleme kontrolünü gerçekleştirmişlerdir [22]. Zeng ve Singh, Lorenz sisteminin durum uzayında belirli bir noktaya getirilebilmesi için adaptif kontrol kuralı elde ederek sistemi kontrol etmişlerdir [23]. Yang, Lorenz sisteminin kontrolü için sliding mode kontrolü önermişlerdir [28]. Yine Alzate ve ark. Lorenz sisteminin kontrolünü sliding mode kullanarak gerçekleştirmişlerdir [29]. Yau ve ark. ise Lorenz sisteminin kontrolü için yine doğrusal olmayan bir yöntem olan sliding mode kontrol ile doğrusal olmayan kontrol işareti üretmişler ve lorenz sistemini denetlenmişlerdir [30]. Chen ve ark.
Lorenz sisteminin denetlenebilmesi için teorik ve numerik olarak backstepping denetleyici tasarlamıştır [31]. Yassen ve ark. ise backstepping yöntemini kullanarak kaotik dinamik sistemlerin kontrol edilebileceğini göstermişlerdir. Ardından bu tekniği, Lorenz, Chen ve Lü kaotik sistemlerinin kontrolünü gerçekleştirmişlerdir [32]. Run-Zi ise kaotik bir sistemin stabilazyonu ve senkronizsyonu için impulsive yöntemini kullanmıştır [33]. Tan ve diğerleri ise kaotik sistemlerin senkronizasyonu için backstepping yöntemini gerçekleştirmişlerdir [34]. Köse ise adaptif kutup yerleştirme ve Sliding mod kullanarak Lorenz sisteminin denetlenmesini karşılaştırmıştır [35]. Pham ve ark. ise 3D Fractional kaotik bir sistemi ele almışlar. $\mathrm{Bu}$ sistemin denge noktası olmamasına rağmen aktif kontrol ile kontrolünü gerçekleştirmişler. Ayrıca bu sistemin devre uygulamasını da gerçekleştirmişler [36]. Longge ise ayrık olan kaotik bir sistemin model öngörülü kontrol senkronizasyonunun gerçekleştirmişlerdir [37]. Park vd yaptığı çalışmada yine ayrık olan kaotik bir sistemin MPC ile kontrolü için genelleştirilmiş model öngörülü kontrolü kullanmışlardır [38]. Mohammadbagheri vd ise Lorenz tipinde bir kaotik sistemin kontrolü için model öngörülü kontrol yöntemini kullanmışlar [20]. Tabatabaei vd ise koatik hibrit bir sistemin kontrolünü explicit model öngörülü kontrol ile gerçekleştirmişlerdir [39].

$\mathrm{Bu}$ çalışmada literatürde doğrusal olmayan sistemlerin kontrolünde kullanılan 4 farklı yöntem ile Lorenz Kaotik sistemi kontrol edilmiştir. $\mathrm{Bu}$ çalışmanın özgünlüğüne bakıldığından Lorenz Koatik sistemi daha önceden integratör içeren model öngörü kontrol yöntemi kontrol edilmemiştir. Ayrıca bu yöntemin yanı sıra Lorenz Kaotik sisteminin $[0,0,0]$ denge noktasına gelmesi için geri beslemeli kontrol, yüksek kazanç kontrolü, yüksek frekans kontrolü ve integratör içeren model öngörülü kontrol yöntemleri kullanılmıştır. Bu çalışmada kullanılan her bir denetleyicinin matematiksel ifadeleri üretilmiş ve Matlab R2018A üzerinde simülasyon çalışmaları yapıllmıştır. Sonuçta ise kullanılan kontrol yöntemlerinin avantaj ve dezavantajları tartışılmıştır.

\section{METOTLAR}

\subsection{Geri Beslemeli Kontrol}

Geri beslemeli doğrusallaştırma genel olarak doğrusal olmayan sistemlerin bir denge noktası etrafında doğrusallaştırılması ve ardından da kapalı çevrim kararlılığını sağlayacak kontrol kuralının belirlenmesi ile gerçekleştirilmektedir. Burada yapılacak sistemin denge 
noktasında etrafinda doğrusallaştırılmasından ziyade sistemi doğrusal bir sistem haline getirecek olan terimlerin kontrol işaretine ilave edilmesidir. $\mathrm{Bu}$ sayede sistem doğrusal bir sistem haline getirilecektir. Ardından kapalı çevrim kararlılığ 1 sağlayan kontrol işareti belirlenecektir. Bunun için ilk olarak kontrol edilmek istenen sisteme $u_{1}, u_{2}$ ve $u_{3}$ kontrol işaretlerinin ilavesi yapılacaktır. $\mathrm{Bu}$ Lorenz sisteminin kontrol işaretlerinin eklenmiş hali Eşitlik (1)'de verilmiştir.

$$
\begin{aligned}
& \dot{x}_{1}=\sigma\left(x_{2}-x_{1}\right)+u_{1} \\
& \dot{x}_{2}=-x_{1} x_{3}+r x_{1}-x_{2}+u_{2} \\
& \dot{x}_{3}=x_{1} x_{2}-b x_{3}+u_{3}
\end{aligned}
$$

Bu sistem Eşitlik (2)'deki doğrusal forma getirilecektir. Bu sistem Eşitlik (2)'deki doğrusal forma getirilecektir.

$\dot{x}=A x+B u$

Doğrusal olmayan sistemin yapısı Eşitlik (3)'te verilmiştir. Doğrusal ve zamanla değişmeyen A matrisi sistem matrisi ve $\mathrm{B}$ kontrol ise matrisidir. Eşitlikte bulunan $\mathrm{a}(\mathrm{x})$ sistemin içinde bulunan doğrusal olmayan terimleri ifade etmektedir. $\mathrm{Bu}$ terimler Eşitlik (4)'de kontrol işaretine ilave edilerek sistemde bulunan doğrusal olmayan terimler yok edilir. Ardından burada $v$ kontrol işareti dizayn edilir.

$\dot{x}=A x+B \gamma(x)[u-a(x)]$

$u=a(x)+\gamma(x)^{-1} v$

$\mathrm{Bu}$ yöntem Lorenz sistemi için genelleştirildiğinde ise kontrol işaretleri Eşitlik (5), Eşitlik (6) ve Eşitlik (7) de gösterildiği gibi elde edilir.

$u_{1}=r x_{1}+v_{1}$

$u_{2}=+x_{1} x_{3}-r x_{1}+x_{2}+x_{3}+v_{2}$

$u_{2}=-x_{1} x_{2}+b x_{3}+v_{3}$

Üretilecek olan kontrol işaretleri ile kapalı çevrim sistemin durum denklemleri Eşitlik (8)'deki gibi olur.

$$
\begin{aligned}
& \dot{x}_{1}=\sigma x_{2}+v_{1} \\
& \dot{x}_{2}=x_{3}+v_{2} \\
& \dot{x}_{3}=-v_{3}
\end{aligned}
$$

Tasarımda sistem kısmen faz kanonik formuna dönüştürülecektir. $\mathrm{Bu}$ nedenle kontrol işaretlerinden $v_{1}, v_{2}$ ve $v_{3}$ Eşitlik (9)'daki gibi tasarlanmıştır. Sistemin olmasını istenen özdeğerlerine yani $\lambda_{1}, \lambda_{2}, \lambda_{3}$ 'lar belirlendikten sonra Eşitlik (9)'da bulunan $k_{1}, k_{2}, k_{3}$ belirlenecektir. $\mathrm{Bu}$ parametreler belirlendikten sonra kontrol işaretleri $u_{1}, u_{2}$ ve $u_{3}$ içinde bulunan $v_{1}, v_{2}$ ve $v_{3}$ işaretleri belirlenmiş olmaktadır.

$$
\begin{array}{lc}
v_{1}= & 0 \\
v_{2}= & 0 \\
v_{3}=k_{1} x_{1}+k_{2} x_{2}+k_{3} x_{3}
\end{array}
$$

Belirlenen kontrol işareti sisteme uygulandığında ise kapalı çevrim sistem matrisi Eşitlik (10)'da verilmiştir.

$$
\left[\begin{array}{c}
\dot{x}_{1} \\
\dot{x}_{2} \\
\dot{x}_{3}
\end{array}\right]=\left[\begin{array}{ccc}
0 & \sigma & 0 \\
0 & 0 & 1 \\
-k_{1} & -k_{2} & -k_{3}
\end{array}\right]\left[\begin{array}{l}
x_{1} \\
x_{2} \\
x_{3}
\end{array}\right]
$$

\subsection{Yüksek Kazanç Kontrolü}

Yüksek kazanç kontrolü Lyapunov kararlılık kriterine dayanan bir denetleyici türüdür. Eşitlik (11)'de verilen doğrusal olmayan sistemin doğrusal ve doğrusal olmayan kısımları birbirilerinden ayrıştırılır. Doğrusal ve zamanla değişmeyen A matrisi sistem matrisi ve B kontrol ise matrisidir. Doğrusal olmayan $a(x)$ ise sistemin içinde bulunan doğrusal olmayan kısımları ifade etmektedir. Sisteme uygulanacak olan kontrol işareti Eşitlik (12)'de verilmiştir.

$\dot{x}=A x+B \gamma(x)[u-a(x)]$

$u=-k x+V_{R}$

Eşitlik (12)'de sisteme uygulanacak olan kontrol işaretindeki $V_{R}$ parametresinin ne olması gerektiği seçilmektedir. Bunun seçimi ise Lyapunov enerji fonksiyonuna göre yapılmaktadır. Sistemin kontrolü için oluşturulan Lyapunov enerji fonksiyonu Eşitlik (13)'de verilmiştir.

$$
\begin{aligned}
V=\frac{1}{2} x_{1}^{2}+\frac{1}{2} x_{2}^{2}+\frac{1}{2} x_{3}^{2} & =x^{T} P x= \\
& =\left[\begin{array}{lll}
x_{1} & x_{1} & x_{1}
\end{array}\right]\left[\begin{array}{lll}
1 & 0 & 0 \\
0 & 1 & 0 \\
0 & 0 & 1
\end{array}\right]\left[\begin{array}{l}
x_{1} \\
x_{1} \\
x_{1}
\end{array}\right]
\end{aligned}
$$

Lyapunov Kararlılığının 2. Kriterini sağlaması için Eşitlik (13)'deki fonksiyonunun içindeki P matrisi pozitif tanımlı olacak şekilde seçilmiştir. Burada seçilen katsayılar enerji fonksiyonunun sürekli $V>0$ yapmaktadır. Diğer bir önemli kriter ise sistemin asimtotik kararlılığı için bu fonksiyonun türevinin sıfırdan küçük olmasıdır. Sıfıra eşit olduğunda ise sadece sistem kararlıdır denilebilmektedir. Aksi halde türevi sıfirdan büyük olduğunda enerjisi artan ve kontrol edilemeyen bir sistem gibi düşünülebilir. $\mathrm{Bu}$ fonksiyonun türevi Eşitlik (14)’te verilmiştir.

$\dot{V}=x_{1} \dot{x}_{1}+x_{2} \dot{x}_{2}+x_{3} \dot{x}_{3}$

Eşitlik (14)'te sistemin durumları yerlerine yazılınca Eşitlik (15) elde edilir.

$$
\begin{aligned}
\dot{V}=x_{1}[ & \left.\sigma\left(x_{2}-x_{1}\right)-k_{11} x_{2}+V_{R 1}\right] \\
& +x_{2}\left[-x_{1} x_{3}+r x_{1}-x_{2}-k_{21} x_{3}+V_{R 2}\right] \\
& +x_{3}\left[x_{1} x_{2}-b x_{3}-k_{31} x_{1}-k_{32} x_{2}-k_{33} x_{3}+V_{R 3}\right]
\end{aligned}
$$

Eşitlik (15) düzenlendiğinde ise Eşitlik (16) elde edilir.

$$
\begin{gathered}
\dot{V}=\left(2 \sigma-k_{11}\right) x_{1} x_{2}+x_{1} V_{R 1}-\left(k_{21}-k_{32}\right) x_{2} x_{3} \\
+x_{2} V_{R 2}-k_{31} x_{1} x_{3}+x_{3} V_{R 3}
\end{gathered}
$$


Eşitlik (16)'daki fonksiyonun $\dot{V} \leq 0$ olması için parametreler Eşitlik (17)'deki gibi düzenlenmiştir.

$\left(2 \sigma-k_{11}\right)=0 \rightarrow k_{11}=2 \sigma$

$\left(k_{21}-k_{32}\right)=0 \rightarrow k_{21}=k_{32}$

$k_{31}=0$

Bu durumda sistemin parametreleri Eşitlik (18)'de yerlerine yazılarak $\dot{V}$ elde edilir.

$$
\dot{V}=x_{1} V_{R 1}+x_{2} V_{R 2}+x_{3} V_{R 3}
$$

Bu fonksiyonun $\dot{V} \leq 0$ sağlaması için $V_{R 1}, V_{R 2}$ ve $V_{R 3}$ Eşitlik (19)'daki gibi seçilmiştir.

$V_{R 1}=-x_{1}$

$V_{R 2}=-x_{2}$

$V_{R 3}=-x_{3}$

\subsection{Yüksek Frekans Kontrolü}

Yüksek frekans kontrol belirli bir duruma göre çok yüksek frekanslarda kontrol işaretini değiştirerek exponansiyel kararlılığ 1 sağlayan ve Lyapunov kararlılık kriterine dayanan bir denetleyici türüdür. Eşitlik (20)'de verilen doğrusal olmayan sistemin doğrusal ve doğrusal olmayan kısımları birbirilerinden ayrıştırılır. Doğrusal ve zamanla değişmeyen A matrisi sistem matrisi ve B kontrol ise matrisidir. Doğrusal olmayan $a(x)$ ise sistemin için bulunan doğrusal olmayan kısımları ifade etmektedir. Sisteme uygulanacak olan kontrol işareti Eşitlik (21)'de verilmiştir.

$$
\begin{aligned}
& \dot{x}=A x+B \gamma(x)[u-a(x)] \\
& u=-k_{1} x+k_{2} \operatorname{sgn}(x)
\end{aligned}
$$

Sistemin kontrolünde yine Lyapunov enerji fonksiyonuna göre yapılmaktadır. Sistemin kontrolü için oluşturulan Lyapunov enerji fonksiyonu aşağıda verilmiştir. $\mathrm{Bu}$ Lyapunov enerji fonksiyonu eğer faz uzayında bir yüzey için tanımlanarak oluşturulan bir ' $s$ ' yüzeyine göre tasarlanmış olsaydı o zaman bu denetleyici kayan kipli kontrol (sliding mode control) olarak adlandırılırdı.

$V=\frac{1}{2} x_{1}^{2}+\frac{1}{2} x_{2}^{2}+\frac{1}{2} x_{3}^{2}$

Lyapunov Kararlılığının 2. Kriterini sağlaması için Eşitlik (22) fonksiyonunun yüksek kazanç kontrolde olduğu gibi $P$ matrisi pozitif tanımlı olacak şekilde seçilmiştir. Burada seçilen katsayılar enerji fonksiyonunun sürekli $V>0$ yapmaktadır. Diğer bir önemli kriter ise sistemin asimtotik kararlılığı için bu fonksiyonun türevinin sıfirdan küçük olmasıdır. Sıfıra eşit olduğunda ise sadece sistem kararlıdır denilebilmektedir. $\mathrm{Bu}$ fonksiyonun türevi Eşitlik (23)'te verilmiştir.

$\dot{V}=x_{1} \dot{x}_{1}+x_{2} x \dot{x}_{2}+x_{3} x \dot{x}_{3}$
Eşitlik (23)'teki sistemin durumları yerlerine yazılınca Eşitlik (24) elde edilir.

$$
\begin{aligned}
\dot{V}=x_{1}\left[\sigma\left(x_{2}-x_{1}\right)\right. & \left.+u_{1}\right] \\
& +x_{2}\left[-x_{1} x_{3}+r x_{1}-x_{2}+u_{2}\right] \\
& +x_{3}\left[x_{1} x_{2}-b x_{3}+u_{3}\right]
\end{aligned}
$$

Eşitlik (24) düzenlendiğinde Eşitlik (25) elde edilir.

$$
\dot{V}=2 \sigma x_{1} x_{2}+x_{1} u_{1}+x_{2} u_{2}+x_{3} u_{3}
$$

Eşitlik 25'teki denklemin $V^{*} \leq 0$ şartını sağlaması için $u_{1}$, $u_{2}$ ve $u_{3}$ Eşitlik 26'daki gibi seçilmiştir.

$$
\begin{aligned}
& u_{1}=-2 \sigma x_{2}-k_{1} \operatorname{sgn}\left(x_{1}\right) \\
& u_{2}=-x_{2}-k_{2} \operatorname{sgn}\left(x_{2}\right) \\
& u_{3}=-x_{3}-k_{3} \operatorname{sgn}\left(x_{3}\right)
\end{aligned}
$$

Kontrol işaretleri Eşitlik (25)'teki yerlerine yazıldığında Eşitlik (27) elde edilir. Bu ifade düzenlediğinde Eşitlik (28) elde edilir. $\mathrm{Bu}$ ifade içindeki $\operatorname{sgn}(x) x$ ifadeleri her zaman pozitif olacağ 1 için bu ifade $|x|$ olarak yerine yazılır. Her bir durum için bu yapıldığında Eşitlik (29) elde edilir.

$$
\begin{aligned}
& \dot{V}=2 \sigma x_{1} x_{2}+x_{1}\left(-2 \sigma x_{2}-k_{1} \operatorname{sgn}\left(x_{1}\right)\right) \\
& +x_{2}\left(-x_{2}-k_{2} \operatorname{sgn}\left(x_{2}\right)\right) \\
& +x_{3}\left(-x_{3}-k_{3} \operatorname{sgn}\left(x_{3}\right)\right) \\
& \dot{V}=-k_{1} \operatorname{sgn}\left(x_{1}\right) x_{1}-x_{2} x_{2}-k_{2} x_{2} \operatorname{sgn}\left(x_{2}\right) \\
& -x_{3} x_{3}-k_{3} \operatorname{sgn}\left(x_{3}\right) x_{3} \\
& \dot{V}=-k_{1}\left|x_{1}\right|-x_{2}^{2}-k_{2}\left|x_{2}\right|-x_{2}^{2}-k_{3}\left|x_{3}\right|
\end{aligned}
$$

\subsection{Model Öngörülü Kontrol}

$\mathrm{Bu}$ bölümde Model Öngörülü kontrol tekniğinin k1saca açıklanacak ve matematiksel formülasyonu verilecektir. MPC kayan ufuk fikrine dayalı olarak geliştirilmiş model tabanlı bir denetleyicidir. Kayan ufuk mantığında her örnekleme anında optimal kontrol probleminin çözümü yapılmaktadır. $\mathrm{Bu}$ işlem aslında referans işareti, sistemin dinamikleri, sistemin ve kontrol işaretinin kısıtlarının içinde bulunduğu bir problemin çözümüdür. Bu ișlem aslında her örnekleme anında yapılarak öngörü ufku boyunca kontrol kontrol kuralı belirlenmektedir. Şekil 1'de gösterildiği gibi sistemin örneklem anındaki $y(t)$ çıkışının bulunan $t$ anı ile $t+N$ anı arasında sistemi beklenen referans işaretine $w(t)$ 'ye ulaştırmak için uygulanan kontrol işaretinin belirlenmesidir. Belirlenen kontrol dizisinin ilk işareti sisteme uygulanarak bir sonraki örnekleme anında yine bu kontrol dizisi sistemin durumlarına göre belirlenerek kontrol kuralının ilk elemanı sisteme uygulanmaktadır. 


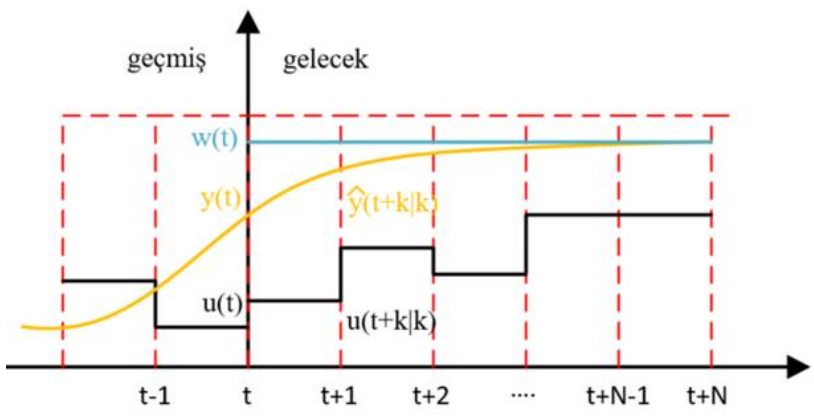

Şekil 1. Model Öngörülü Kontrol Şeması

Model öngörülü kontrol tekniğinin kalbi olan model kullanılarak öngörü ufku boyunca örnekleme anında sisteme uygulanması gereken kontrol işaretini belirlemektedir. Doğrusal olmayan sistemlerde ise bu kontrol tekniği uygulanırken sistemin çalıştığı bölgeye göre doğrusallaştırılmış modeli kullanılarak yine sistem kontrol edilmektedir.

Lorenz kaotik sisteminin sürekli zamandaki sistem dinamiği $\dot{x}=f(x, u)$ formundadır. $\mathrm{Bu}$ sistemin MPC ile kontrol edilebilmesi için belirli bir çalışma noktası etrafında sistemin doğrusallaştırılması gerekmektedir. Doğrusallaştırılan sistemin örnekleme zamanında göre ayrıklaştırılması gerekmektedir. Doğrusallaştırılan ve ayrıklaştırılan sistemin MPC ile kontrolü gerçekleştirilmiştir. Ayrıca sistemin daha iyi kontrol edilebilmesi yani referans işaretini daha iyi takip edilebilmesi için sistemin çıkışlarına yani durumlarına integratör ilave edilmiştir. $\mathrm{Bu}$ durumdan kontrol edilecek olan kaotik sistemin durum uzayındaki ifadesi Eşitlik (30)'daki gibi olmaktadır.

$\hat{x}_{1}=\sigma\left(x_{2}-x_{1}\right)+u_{1}$

$\hat{x}_{2}=-x_{1} x_{3}+r x_{1}-x_{2}+u_{2}$

$\hat{x}_{3}=x_{1} x_{2}-b x_{3}+u_{3}$

$\hat{x}_{1}=-x_{2}$

$\hat{x}_{2}=-x_{2}$

$\hat{x}_{3}=-x_{3}$

$\mathrm{Bu}$ sistemin yapısı Eşitlik (31)'deki gibi ifade edilebilir.

$\dot{x}=f(x, u, t)$

$y=g(x, u, t)$

Doğrusal olmayan bu Lorenz sistemi her örnekleme anında doğrusallaştırılacaktır. Eşitlik (32)'de verilen Jakobien matirisi ile sistemin çalıştığı yere göre doğrusallaştırılmış sistem matrisleri elde edilir.

$A=\frac{\partial f}{\partial x} \quad B=\frac{\partial f}{\partial u}$

$C=\frac{\partial g}{\partial x} \quad D=\frac{\partial g}{\partial u}$

Doğrusallaştırılmış olan sistemin ayrıklaştırılması yapılacaktır. Sistemin her örnekleme anında tekrardan oluşturulacak olan ayrık modeli eşitlik ise Eşitlik (33)'de verilmiştir. Buradaki sistem matrisi ve kontrol matrisi için Eşitlik (34)'de verilen basit ayrıklaştırma yöntemi kullanılmıştır. Burada sistem matrisi $x(k)$ anındaki sistemin durumlarına göre ve $u(k-1)$ anında sisteme uygulanan kontrol işaretine göre elde edilmektedir.

$x(k+1)=A_{d} x(k)+B_{d} u(k)$

$A_{d}=\left.\left(I+A T_{s}\right)\right|_{x=x(k), u=u(k-1)}$

$B_{d}=\left.B T_{s}\right|_{x=x(k), u=u(k-1)}$

$x(k) \in R^{n} \quad u(k) \in R^{m}$

Kabul olarak sistemin her örnekleme anında hem kontroledilebilir olduğu hem de gözlenebilir olduğu varsayımı yapılmıştır. Amaç fonksiyonu öngörü ufku boyunca sistemin davranışını belirleyecek olan optimal kontrol problemidir. $\mathrm{Bu}$ fonksiyon Eşitlik (35)'de verilmiştir. $\mathrm{Bu}$ optimal kontrol problemi, $x(k)$ ve $u(k)$ matrisleri ile çok değişkenli optimizasyon problemi haline dönüştürülür. $\mathrm{Bu}$ problemin çözümünde $\mathrm{QP}, \mathrm{SQP}$ algoritmaları kullanılarak çözüldükten sonra, mevcut örnekleme zamanı için öngörü ufku boyunca kontrol kuralı elde edilir. Bu kontrol ufkunun sadece ilk elemanı sisteme uygulanır. Bir sonraki örnekleme zamanında, öngörü ve kontrol ufku boyunca yeni kontrol kuralı üretmek için bu optimizasyon süreci tekrarlanır

$\underset{\left(x_{k}, u_{k}\right)}{\min J}=\sum_{k=0}^{N} x_{k}^{T} Q x_{k}+\sum_{k=0}^{M} u_{k}^{T} R u_{k}$

\section{SİMÜLASYON ÇALIŞMASI}

$\mathrm{Bu}$ bölümde Lorenz sisteminin kontrolü geri beslemeli kontrolü, yüksek kazanç kontrolü, yüksek frekans kontrolü ve model öngörülü kontrol yöntemlerinin simülasyonları gerçekleştirilmiş ve sonuçları verilmiştir. $\mathrm{Bu}$ sistemlerin simülasyonları için MATLAB Simulink R2018A programı kullanılmıştır.

Eşitlik (1)'de bulunan sistemin $\sigma=10, r=\frac{8}{3}, b=28$ olarak verilmiştir.

Geri beslemeli kontrol yönteminde sistemin denetlenmesi için denetleyicinin parametreleri belirlenmelidir. Bunun için kapalı çevrimde kullanılacak olan sistem kutupların keyfi olarak $\lambda_{1}=-1, \lambda_{2}=-2, \lambda_{3}=-3$ olmas1 istenmektedir. $\mathrm{Bu}$ başka bir performans olarak seçilebilirdi. Buna göre atanan kutupların bu değerleri de olabilmesi için sistemin kapalı çevrimde bu kutupları sağlayacak olan k parametreleri $k_{1}=\frac{6}{11}, k_{2}=11$ ve $k_{3}=6$ 'dir. $\mathrm{Bu}$ yöntemde kontrol işareti Eşitlik (36)'daki gibi oluşturulmuştur. Parametreler Eşitlik (37)'de yerlerine yazılmıştır.

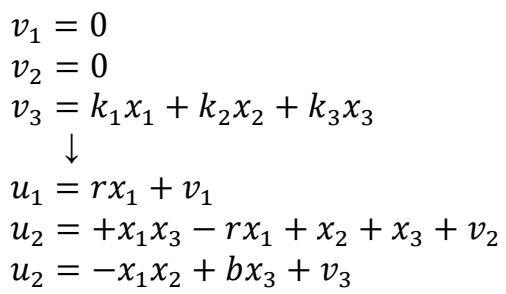


$u_{1}=r x_{1}$

$u_{2}=+x_{1} x_{3}-r x_{1}+x_{2}+x_{3}$

$u_{2}=-x_{1} x_{2}+b x_{3}+\left(\frac{6}{11}\right) x_{1}+(11) x_{2}+(11) x_{3}$

Kutup atama yöntemi ile tasarlanan denetleyicinin kapalı çevrim yapısı Eşitlik (38)'deki gibi olmaktadır

$$
\begin{array}{lc}
\dot{x}_{1}= & \sigma x_{2} \\
\dot{x}_{2}= & x_{3} \\
\dot{x}_{3}= & -\left(\frac{6}{11}\right) x_{1}+(11) x_{2}+(11) x_{3}
\end{array}
$$

Tasarlanan sistemin kapalı yapısı Şekil 2'de verilmiştir. Sistem yanıtı ise Şekil 3'te verilmiştir. Sistem ilk başta kaotik davranış sergilemektedir. 50 saniye sonra tasarlanan denetleyici aktif olmaktadır. Görüldüğü gibi sistem daha önceden tasarlanan $[0,0,0]$ noktasına denetleyici taşıyabilmektedir. Bu tasarım farklı bir denge noktası için yapılsaydı sistem belirlenen denge noktasına taşınabilirdir. Ayrıca sistemin kontrol işaretleri Şekil 4'te verilmiştir. Ardından literatürde yapılan bir çalışma ile karşılaştırma amacı ile [27]'de yapılan çalışmada elde edilen kontrol kuralı ile elde edilen sistemin durumları Şekil 5'de verilirken kontrol kuralı Şekil 3'de verilmiştir. Sistem yanıtları incelendiğinde sistemin denge noktasında sürekli salınım yaptığı ve performansının düşük olduğu görülmektedir. Bunun nedeni tüm durumları tek bir kontrol işareti ile kontrol edilmesidir. Tüm durumlar istenen performans Şekil 4'deki gibi sağlanabilmektedir. Ayrıca keyfi olarak seçilen kutuplar değiştirilerek sistemin performansı istenildiği gibi ayarlanabilir. Ayrıca Şekil 6'da görüldüğü üzere sisteme kararlı hale geçemediği için kontrol işareti sürekli olarak salınım yapmaktadır. Bu sonuçtan görüldüğü üzere sistemin tüm durumları kontrol edildiğinde istenen performans geri beslemeli kontrol ile literatürdeki bazı çalışmalardan daha iyi bir şekilde elde edilebilmektedir.

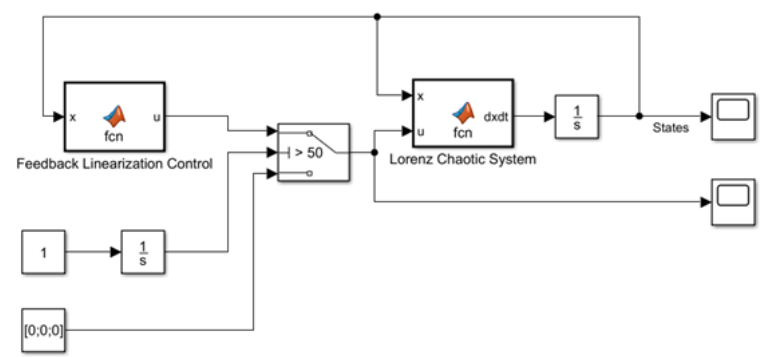

Şekil 2. Lorenz kaotik sisteminin geri besleme ile kontrolünün blok diyagramı

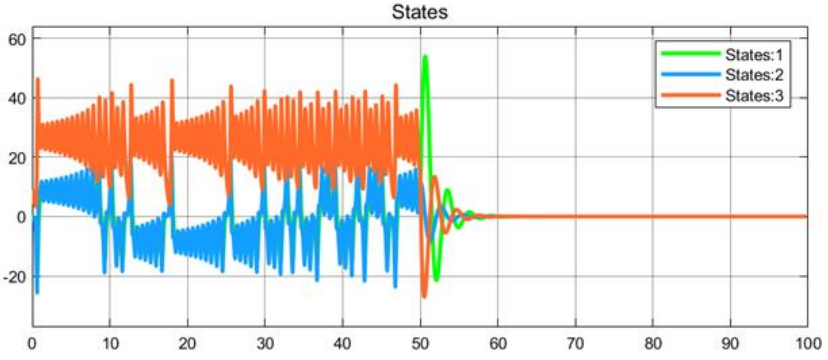

Şekil 3. Lorenz kaotik sisteminin geri beslemeli kontrol ile elde edilen sistemin durumları

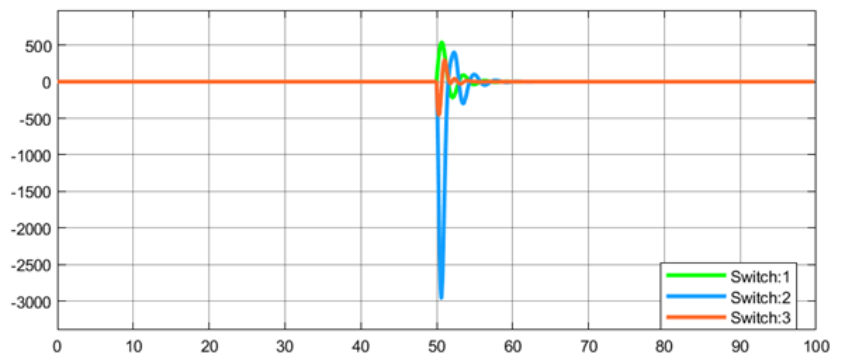

Şekil 4. Lorenz kaotik sisteminin geri beslemeli kontrol ile elde edilen kontrol işaretleri

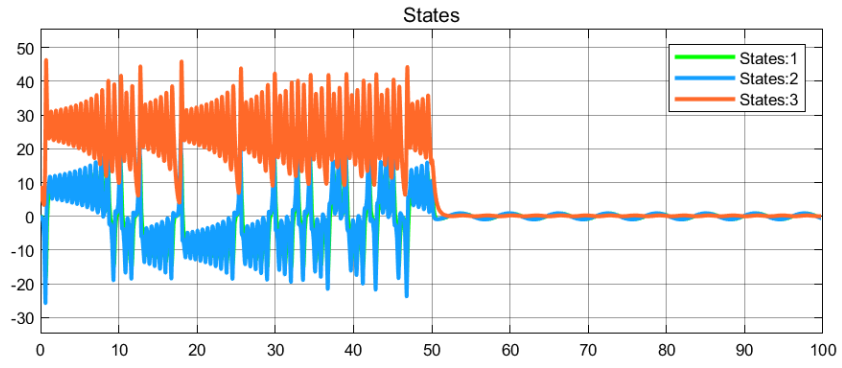

Şekil 5. Lorenz kaotik sisteminin literatürdeki geri beslemeli kontrol kuralı ile elde edilen sistemin durumları

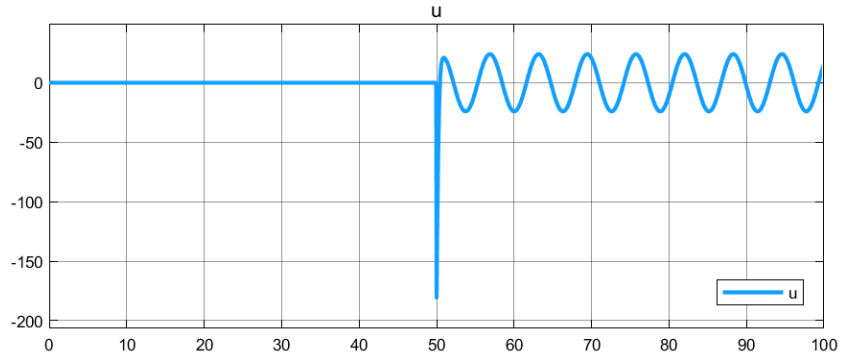

Şekil 6. Lorenz kaotik sisteminin literatürdeki geri beslemeli kontrol kuralı ile elde edilen kontrol işareti

Yüksek Kazanç kontrolü ile Lorenz sistemi kontrol edilmek istendiğinde sisteme uygulanacak olan kontrol işaretleri Eşitlik (19)'da verilmişti. Bu kontrol işaretinin uygulanabilmesi için Eşitlik (18)'de verilen fonksiyonun $\dot{V} \leq 0$ yapmaktadır. Bu kontrol kuralındaki parametrelerin belirlenmesi Eşitlik (17)'de yapılmıştı. $k_{11}=2 \sigma, k_{21}=$ $k_{32}, k_{21}=k_{32}$ ve $k_{31}=0$ seçildiği için Eşitlik (18)'deki denklem $\dot{V} \leq 0$ şartını sağlamaktadır. Tasarlanan sistemin kapalı yapısı Şekil 7'de verilmiştir. Sistem yanıtı ise Şekil 8'de verilmiştir. Sistem ilk başta kaotik davranış sergilemektedir. 50 saniye sonra sistem için tasarlanan denetleyici aktif olmaktadır. Görüldüğü gibi sistem daha önceden tasarlanan $[0,0,0]$ noktasina denetleyici taşıyabilmektedir. Bu tasarım farklı bir denge noktası için yapılsaydı sistem belirlenen denge noktasına taşınabilirdir. Ayrıca sistemin kontrol işaretleri Şekil 8'de verilmiştir. Literatürdeki Qiao ve ark. yaptığı çalışmada yüksek kazanca benzer bir yöntem ile denetlenen Lorenz sistemi tek durum üzerinden kontrol edilmiştir [26]. Qiao ve ark. önerdikleri denetleyici ile kontrol edilen sistemin durumları Şekil 10'da verilirken, sistemi kontrol eden kontrol işareti Şekil 11'da verilmiştir. Görüldüğü üzere Şekil 8 ve Şekil 10 karşılaştırıldığında sadece beklenen referans işaretine Şekil 
8'de daha hılı geldiği ve Şekil 10'daki gibi sistemin 2. durumundaki (mavi renkli) kadar aşım yapmadığ görülmektedir.

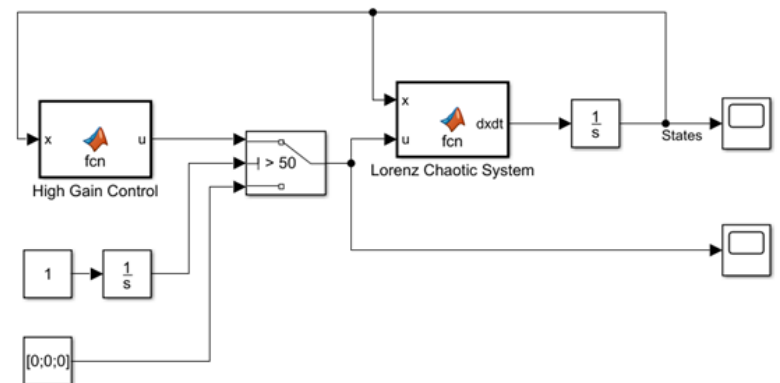

Şekil 7. Lorenz kaotik sisteminin yüksek kazanç ile kontrolünün blok diyagramı

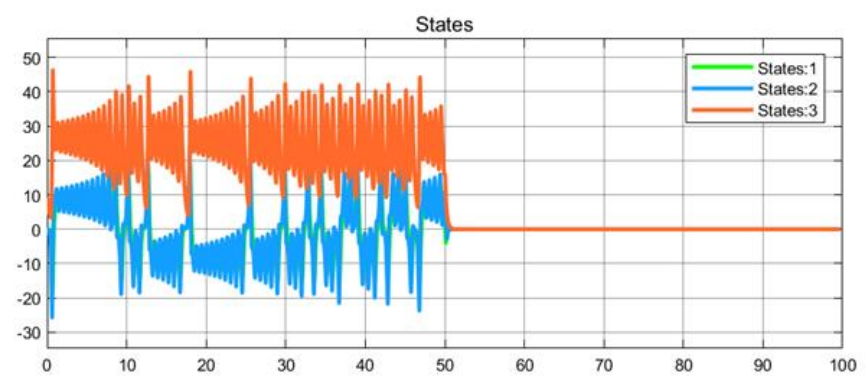

Şekil 8. Lorenz kaotik sisteminin yüksek kazanç kontrolü ile elde edilen sistemin durumları

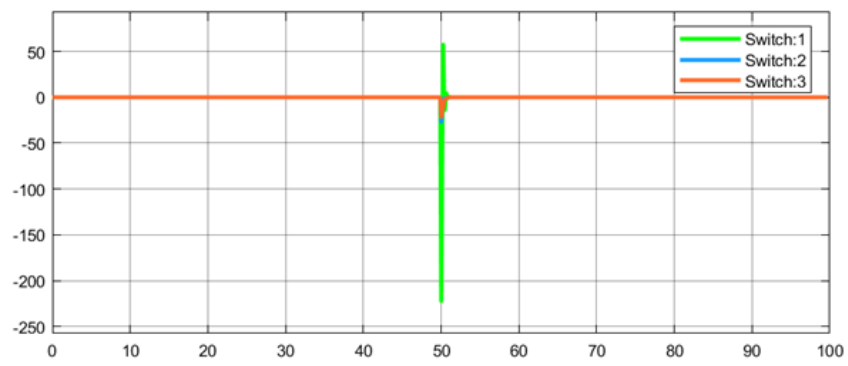

Şekil 9. Lorenz kaotik sisteminin yüksek kazanç kontrolü ile elde edilen kontrol ișaretleri

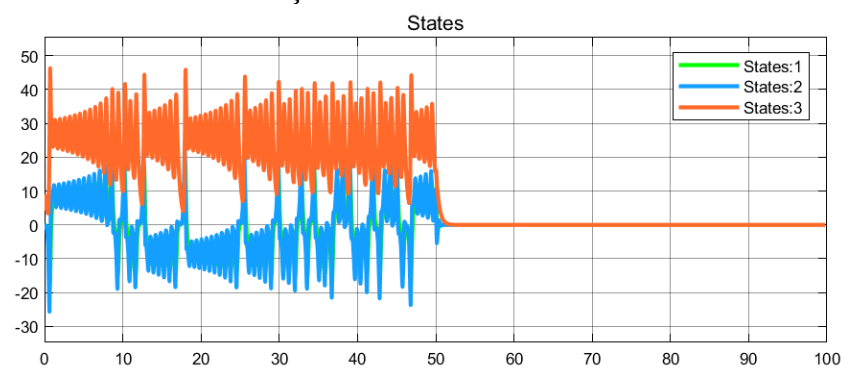

Şekil 10. Lorenz kaotik sisteminin literatürdeki yüksek kazanç kontrol kuralı ile elde edilen sistemin durumları

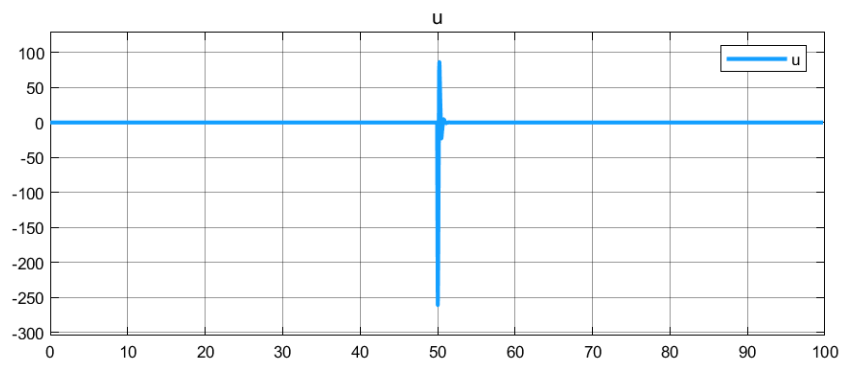

Şekil 11. Lorenz kaotik sisteminin literatürdeki yüksek kazanç kontrolü kuralı ile elde edilen kontrol işareti Yüksek frekans kontrolü ile Lorenz sistemi kontrol edilmek istendiğinde sisteme uygulanacak olan kontrol işaretleri Eşitlik (26)'da verilmişti. Bu kontrol işaretinin uygulanabilmesi için Eşitlik (39)'da verilen fonksiyonun $\dot{V} \leq 0$ olması gereklidir. Bu şartlar $k_{1}, k_{2}, k_{3} \geq 0$ olduğunda sağlandığ 1 için keyfi olarak $k_{1}=1, k_{2}=1$ ve $k_{3}=1$ olarak seçilmiştir. Dolayısıyla uygulanacak olan kontrol işareti Eşitlik (40)'da verilmiştir.

$$
\begin{aligned}
& \dot{V}=-k_{1}\left|x_{1}\right|-x_{2}^{2}-k_{2}\left|x_{2}\right|-x_{2}^{2}-k_{3}\left|x_{3}\right| \leq 0 \\
& \forall x \neq 0 \text { ve } k_{1}, k_{2}, k_{3} \geq 0 \\
& u_{1}=-2 \sigma x_{2}-k_{1} \operatorname{sgn}\left(x_{1}\right) \\
& u_{2}=-x_{2}-k_{2} \operatorname{sgn}\left(x_{2}\right) \\
& u_{3}=-x_{3}-k_{3} \operatorname{sgn}\left(x_{3}\right) \\
& \downarrow \\
& u_{1}=-2(10) x_{2}-(1) \operatorname{sgn}\left(x_{1}\right) \\
& u_{2}=-x_{2}-(1) \operatorname{sgn}\left(x_{2}\right) \\
& u_{3}=-x_{3}-(1) \operatorname{sgn}\left(x_{3}\right)
\end{aligned}
$$

Tasarlanan sistemin blok yapısı Şekil 12'de verilmiştir. Sistem yanıtı ise Şekil 13'de verilmiştir. Sistem ilk başta kaotik davranış sergilemektedir. 50 saniye sonra tasarlanan denetleyici aktif olmaktadır. Görüldüğü gibi sistem daha önceden tasarlanan $[0,0,0]$ noktasına denetleyici taşıyabilmektedir. Bu tasarım farklı bir denge noktası için yapılsaydı sistem belirlenen denge noktasına taşınabilirdir. Ayrıca sistemin kontrol işaretleri Şekil 14'de verilmiştir. Ardından literatürde yapılan bir çalışma ile karşılaştırma amacı ile [35]'de yapılan çalışmada elde edilen kontrol kuralı ile elde edilen sistemin durumları Şekil 15'de verilirken kontrol kuralı Şekil 16'da verilmiştir. Şekil 15'de görüldüğü üzere sistem durumlarından 2. durum (mavi renkli) ufakta olsa belirli bir kararlı durum hatası ile sürekli hale geçmektedir. Ayrıca kontrolcü bu kararlı durum hatasını sürekli rejimde düzeltmemektedir. Bu sonuçtan görüldüğü üzere sistemin tüm durumları kontrol edildiğinde sistem istenen denge noktasına literatürdeki bazı çalışmalardan daha iyi bir şekilde geldiği görülmüştür. 


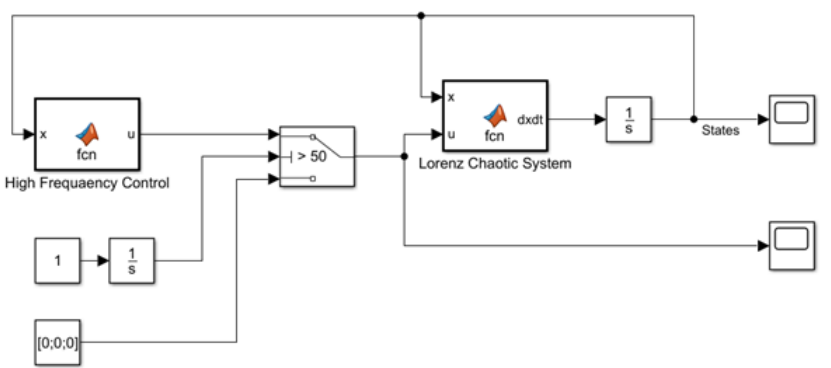

Şekil 12. Lorenz kaotik sisteminin yüksek frekans ile kontrolünün blok diyagramı

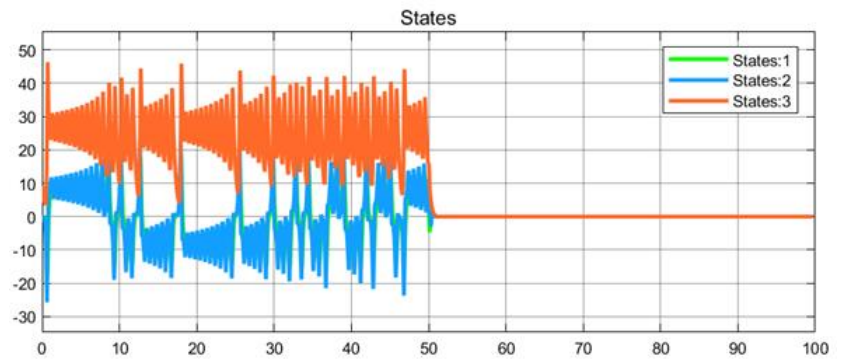

Şekil 13. Lorenz kaotik sisteminin yüksek frekans kontrolü ile elde edilen sistemin durumları

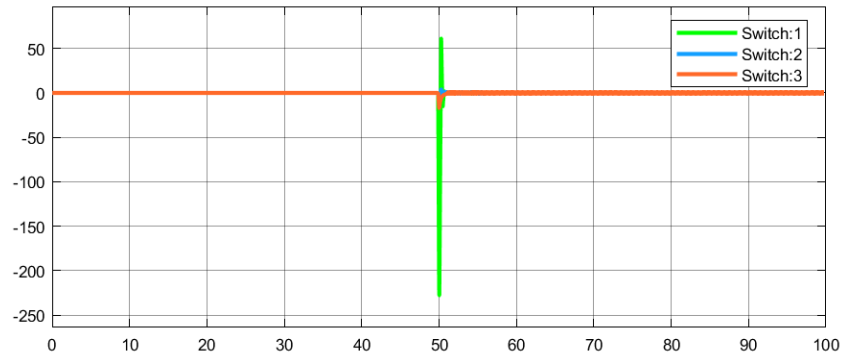

Şekil 14. Lorenz kaotik sisteminin yüksek frekans kontrolü ile elde edilen kontrol ișaretleri

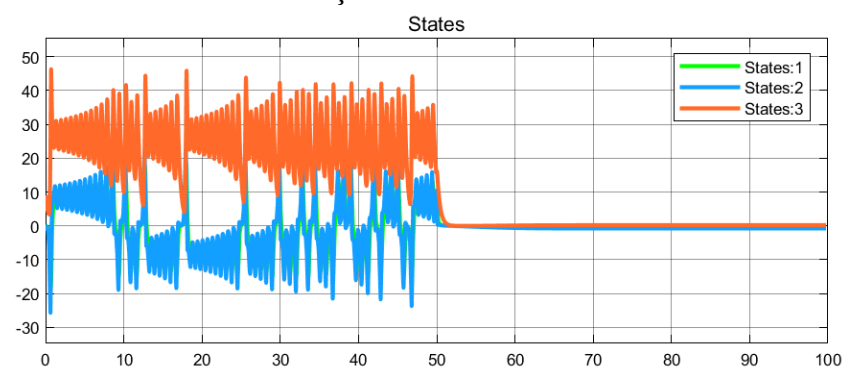

Şekil 15. Lorenz kaotik sisteminin literatürdeki yüksek frekans kontrol kuralı ile elde edilen sistemin durumları

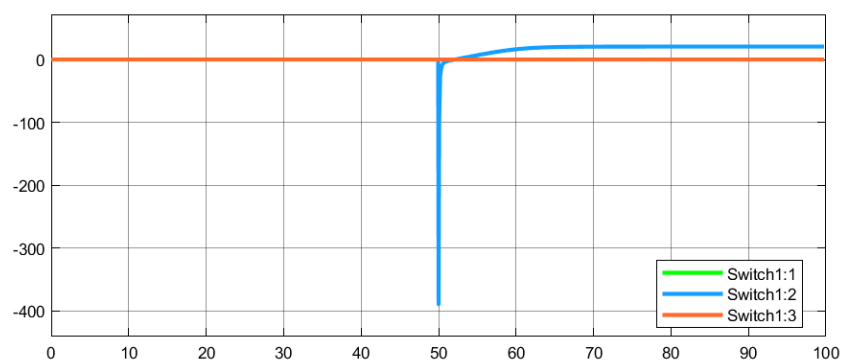

Şekil 16. Lorenz kaotik sisteminin literatürdeki yüksek frekans kontrol kuralı ile elde edilen kontrol işareti
Lorenz sisteminin kontrol edilebilmesi için model öngörülü kontrol denetleyicinin öngörü ufku parametresi $(N) 100$ ve kontrol ufku parametresi $(M)$ 'si 1 seçilmiştir. Ayrıca sistemde kullanılacak olan $\mathrm{P}$ ve $\mathrm{Q}$ matrisleri sistemin davranışını büyük oranda etkilemektedir. Lakin burada yine $P$ matrisinin pozitif tanımlı olabilmesi için diyagonal olarak köşegen elemanları 1 olan $6 x 6$ boyutunda bir matris seçilmiştir. Ayrıca Q matrisi yine kontrol işaretinin ağırlıklarını belirlediği için bunlarında ağırlıkları 1 olarak seçilmiştir. Bu matrisler Eşitlik (41)'de verilmiştir. Sistem kaotik olarak hareket ettiği için denetleyicide kullanılan örnekleme zamanı $\left(T_{S}\right) 1 m s$ olarak seçilmiștir.

$P=\left[\begin{array}{llllll}1 & 0 & 0 & 0 & 0 & 0 \\ 0 & 1 & 0 & 0 & 0 & 0 \\ 0 & 0 & 1 & 0 & 0 & 0 \\ 0 & 0 & 0 & 1 & 0 & 0 \\ 0 & 0 & 0 & 0 & 1 & 0 \\ 0 & 0 & 0 & 0 & 0 & 1\end{array}\right] \quad Q=\left[\begin{array}{lll}1 & 0 & 0 \\ 0 & 1 & 0 \\ 0 & 0 & 1 \\ 0 & 0 & 0 \\ 0 & 0 & 0 \\ 0 & 0 & 0\end{array}\right]$

Tasarlanan sistemin yapısı Şekil 16'da verilmiştir. Sistem yanıtı ise Şekil 17'de verilmiştir. Sistem ilk başta kaotik davranış sergilemektedir. 50 saniye sonra tasarlanan denetleyici aktif olmaktadır. Görüldüğ̈̈ gibi sistem daha önceden tasarlanan $[0,0,0]$ noktasina denetleyici taşıyabilmektedir. Bu tasarım farklı bir denge noktası için yapılsaydı sistem belirlenen denge noktasına taşınabilirdir. Ayrıca sistemin kontrol işaretleri Şekil 18'de verilmiştir.

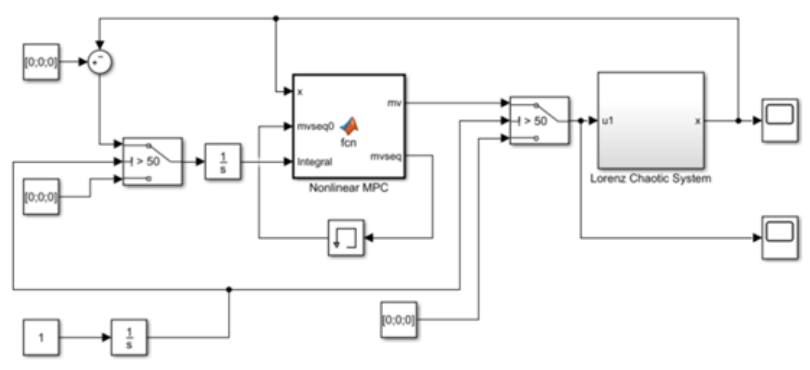

Şekil 16. Lorenz kaotik sisteminin model öngörülü kontrol ile kontrolünün blok diyagram

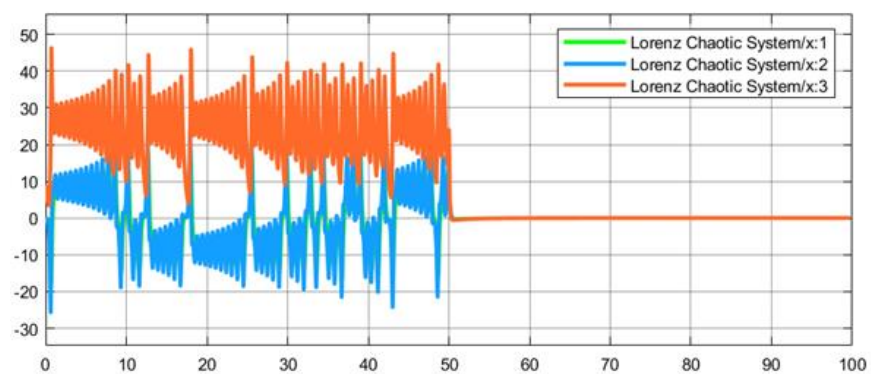

Şekil 17. Lorenz kaotik sisteminin model öngörülü kontrol kontrolü ile elde edilen sistemin durumları 


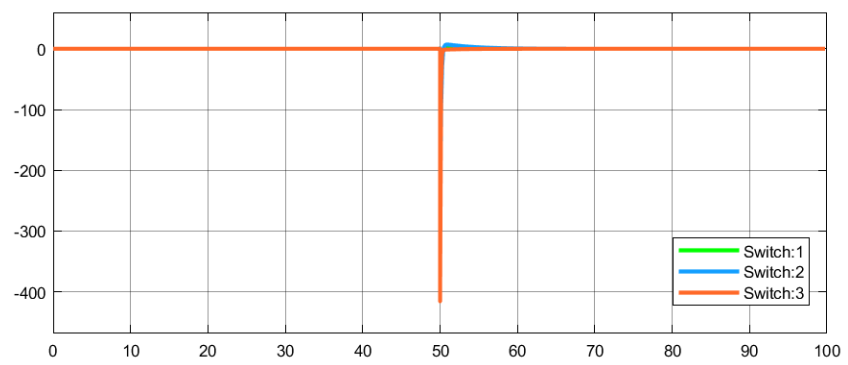

Şekil 18. Lorenz kaotik sisteminin model öngörülü kontrol kontrolü ile elde edilen kontrol işaretleri

\section{SONUÇ}

Yapılan bu çalışmada kaotik Lorenz sisteminin kontrolünde geri beslemeli, yüksek kazanç, yüksek frekans ve model öngörülü kontrol teknikleri kullanılarak kontrol edilmiştir. $\mathrm{Bu}$ yöntemlerden geri beslemeli kontrol, yüksek kazanç kontrolden farklı olarak geri beslemeli kontrolünde; sistemin çalışacağ1 bölgeye göre sistemin durağan sabit bir sistem matrisinin olmasıdır. Tüm doğrusal olmayan yapılar kontrol işareti içerisine gömülerek sistemin doğrusal yapısı elde edilmiştir. Ardından sistem matrisine göre sisteme denetleyici tasarımı yapılarak kapalı çevrim kararlılığı garanti altına alınmaktadır. Ama yüksek kazanç ve yüksek frekans kontrolünde sistemin kararlılığını belirleyen şey Lyapunov fonksiyonu yani enerji fonksiyonudur. Denetleyici oluşturulan bu fonksiyona ve gerekli şartlarına göre tasarlanmaktadır. Bu nedenle tüm parametrelerin bildiği farz edilerek tasarlanan geri beslemeli kontrol, parametrelere duyarlı bir kontrol tekniğidir. Buna mukabil yüksek kazanç kontrol, yüksek frekans kontrol ve model öngörülü kontrol teknikleri enerji fonksiyonları üzerinden tasarlandıkları için daha dayanıklı denetleyicilerdir. Buna mukabil tüm sonuçlar incelendiğinde sistemin kontrol edilmesinde en iyi performansı hem yüksek kazanç kontrolü hem de model öngörülü kontrol göstermiştir. Lakin yüksek kazanç kontrolünde; optimal bir kontrol işaretini üretmekten ziyade, enerji fonksiyonunu minimum yapması istenen bir kontrol kuralının üretilmesi amaçlanmaktadır. Yani sistemin performansından ziyade kararlığın sağlanacağı bir kontrol işaretinin üretilmesi esasına dayanmaktadır. Buna mukabil model öngörülü kontrolde amaç fonksiyonu; hem durumların hem de kontrol işaretinin karesel hatalarının toplamını içeren bir yapısı bulunmaktadır. Dolayısıyla optimal kontrol açısından bakıldığında model öngörülü kontrol belirlenen performans kriterini her örnekleme anında optimize ettiğinden sisteme uygulanacak olan optimal kontrol işaretinin üretilmesini ve sistemi istenen optimal noktaya taşınmasını en iyi performansta yapmaktadır. Bunun yanında bu çalışmada ele alınmamasına rağmen ama model öngörülü kontrolün avantajlarına bakıldığında çok girişliçıkışlı sistemlere rahatlıkla uygulanabildiği gibi sistem kısıtlarının da işin içine kolaylıkla katabilmektedir. Yüksek kazanç kontrolünde sistem modelinde belirsizlikler olduğu durumlarda gerekli kontrol işaretini aktuatör üretmeyebilmekte ve saturasyona neden olabilmektedir. Yüksek frekans kontrolü bunun üstesinden gelmekte fakat belirli bir durağan noktada çok yüksek frekanslarda anahtarlama yapılması gerektiğinden bu sisteme zarar verebilmektedir veya aktuatörün zarar görmesine neden olabilmektedir. Model öngörülü kontrolde bu gibi durumların üstesinden rahatlıkla gelebilmektedir. Ama Model öngörülü kontrolde ise diğer denetleyicilere nazaran her örnekleme anında çok yüksek bir hesaplama işlemi yapılmaktadır. $\mathrm{Bu}$ işlemlerin örnekleme süresi içinde tamamlanması gerekmektedir. Eğer kontrol işareti örnekleme zamanı içinde üretilemezse bu sistemin kontrol edilememesine neden olabilmektedir. Bunun üstesinden gelinebilmesi içinde model öngörülü kontrol için literatürde genellikle explicit denilen daha önceden oluşturulmuş lookup tablosu yada eğitilmiş model yapıları kullanılmaktadır. İlerleyen çalışmalarda ise bu yöntemlerin yanı sıra sliding mode ve backstepping, adaptif sliding, fuzzy sliding adaptif backstepping, fuzzy backstepping yöntemleri de kullanılacak olup farklı kaotik sistemlerin kontrolü ve senkronizasyonları gerçekleştirilecektir.

\section{KAYNAKÇA}

[1] V. G. Ivancevic and T. T. Ivancevic, Highdimensional chaotic and attractor systems: a comprehensive introduction. Springer Science \& Business Media, 2007.

[2] W. J. Burroughs, Climate change in prehistory: The end of the reign of chaos. Cambridge University Press, 2005. [3] S. Solhjoo, A. M. Nasrabadi, and M. R. H. Golpayegani, "Classification of chaotic signals using HMM classifiers: EEG-based mental task classification," in 2005 13th European Signal Processing Conference, IEEE, pp. 14, 2005.

[4] G.-Q. Wu et al., "Chaotic signatures of heart rate variability and its power spectrum in health, aging and heart failure," PloS one, vol. 4, no. 2, 2009.

[5] D. S. Gutzler, "Ecological Climatology: Concepts and Applications," ed: JSTOR, 2003.

[6] R. Steinitz, "Music, maths \& chaos," The Musical Times, vol. 137, no. 1837, pp. 14-20, 1996.

[7] J. Harley, "Generative processes in algorithmic composition: Chaos and music," Leonardo, vol. 28, no. 3, pp. 221-224, 1995.

[8] M. Baptista, "Cryptography with chaos," Physics letters A, vol. 240, no. 1-2, pp. 50-54, 1998.

[9] B. Ramakrishnan et al. "Infinite attractors in a chaotic circuit with exponential memristor and Josephson junction resonator," AEU-International Journal of Electronics and Communications, vol. 123, 2020.

[10] A. Akgul, I. M. Moroz and A. Durdu. "A novel data hiding method by using a chaotic system without equilibrium points," Modern Physics Letters B, vol. 33, no. 29, 2019.

[11] M. Sharafi, F. Fotouhi-Ghazvini, M. Shirali, and M. Ghassemian, "A low power cryptography solution based on chaos theory in wireless sensor nodes," IEEE Access, vol. 7, pp. 8737-8753, 2009.

[12] S. Cicek, U. E. Kocamaz and Y. Uyaroğlu. "Secure chaotic communication with jerk chaotic system using sliding mode control method and its real circuit implementation," Iranian Journal of Science and 
Technology, Transactions of Electrical Engineering, vol. 43, no. 3, pp. 687-698, 2019.

[13] J. S. Khan and J. Ahmad, "Chaos based efficient selective image encryption," Multidimensional Systems and Signal Processing, vol. 30, no. 2, pp. 943-961, 2019.

[14] A. Gökyildirim, Y. Uyaroglu and I. Pehlivan. "A Weak Signal Detection Application Based on Hyperchaotic Lorenz System." Tehnički vjesnik, vol. 25, no. 3, pp. 701708, 2018.

[15] A. Gökyildirim, Y. Uyaroglu and I. Pehlivan. "A novel chaotic attractor and its weak signal detection application." Optik, vol. 127, no. 19, 2016.

[16] M. E. Cimen, Z. B. Garip, M. A. Pala, A. F. Boz, and A. Akgul, "Modelling of a Chaotic System Motion in Video with Artifficial Neural Networks," Chaos Theory and Applications, vol. 1, no. 1, pp. 38-50.

[17] M. E. Çimen, S. Kaçar, E. Güleryüz, B. Gürevin, and A. Akgül, "Kaotik bir hareket videosunun yapay sinir ağları ile modellenmesi," Balıkesir Üniversitesi Fen Bilimleri Enstitüsü Dergisi, vol. 20, no. 3, pp. 23-35, 2018.

[18] E. OTT, C. Grebogi, and J. Yorke, "Physical Review etters 64, 1196\} 1199," Controlling chaos, 1990.

[19] T. L. V. J. YU, "Control of a Chaotic System," Dynamics and Control vol. 1, pp. 35-52, 1990.

[20] A. Mohammadbagheri and M. Yaghoobi, "LorenzType Chaotic attitude control of satellite through predictive control," in 2011 Third International Conference on Computational Intelligence, Modelling \& Simulation, 2011: IEEE, pp. 147-152.

[21] Lu, Xia. "A Financial Chaotic System Control Method Based on Intermittent Controller." Mathematical Problems in Engineering, 2020, doi:10.1155/2020/5810707. [22] T. Yeap and N. Ahmed, "Feedback control of chaotic systems," Dynamics and Control, vol. 4, no. 1, pp. 97-114, 1994.

[23] Y. Zeng and S. N. Singh, "Adaptive control of chaos in Lorenz system," Dynamics and Control, vol. 7, no. 2, pp. 143-154, 1997.

[24] S. Emiroglu ve Y. Uyaroğlu. "T Kaotik Sisteminin Geri Besleme ile Kontrolü", Iğdır Üniversitesi Fen Bilimleri Enstitüsü Dergisi, vol. 10, no. 3, pp, 1649-1658, 2020.

[25] H. Kizmaz, U. E. Kocamaz and Y. Uyaroğlu. "Control of memristor-based simplest chaotic circuit with one-state controllers." Journal of Circuits, Systems and Computers, vol. 28, no. 1, 2019.

[26] Z. Qiao and J. Cheng. "A novel linear feedback control approach of Lorenz chaotic system." 2006 International Conference on Computational Inteligence for Modelling Control and Automation and International Conference on Intelligent Agents Web Technologies and International Commerce (CIMCA'06). IEEE, 2006.

[27] F. Gao et al. "Nonlinear feedback control for the
Lorenz system." Dynamics and control, vol. 11, no. 1, 2001. [28] S.-K. Y. C.-L. C. H.-T. Yau, "Control of chaos in Lorenz system," Chaos, Solitons and Fractals vol. 13, pp. 767-780, (2002).

[29] P. P. Cardenas Alzate, G. C. Velez, and F. Mesa, "Chaos control for the Lorenz system," Advanced Studies in Theoretical Physics, vol. 12, no. 4, pp. 181-188, 2018, doi: 10.12988/astp.2018.8413.

[30] H.-T. Yau and J.-J. Yan, "Design of sliding mode controller for Lorenz chaotic system with nonlinear input," Chaos, Solitons \& Fractals, vol. 19, no. 4, pp. 891-898, 2004, doi: 10.1016/s0960-0779(03)00255-8.

[31] M. Chen, D. Zhou, and Y. Shang, "Nonlinear feedback control of Lorenz system," Chaos, Solitons \& Fractals, vol. 21, no. 2, pp. 295-304, 2004, doi: 10.1016/j.chaos.2003.12.066.

[32] M. T. Yassen, "Chaos control of chaotic dynamical systems using backstepping design," Chaos, Solitons \& Fractals, vol. 27, no. 2, pp. 537-548, 2006, doi: 10.1016/j.chaos.2005.03.046.

[33] L. Run-Zi, "Impulsive control and synchronization of a new chaotic system," Physics Letters A, vol. 372, no. 5, pp. 648-653, 2008, doi: 10.1016/j.physleta.2007.08.010.

[34] E. Daş, "Güdümlü Bir Mühimmat Kanatçık Tahrik Sistemi İçin İki Döngülü Kontrol Sistemi Tasarımı,” Fen Bilimleri Enstitüsü, İTÜ, 2014.

[35] E. Köse and A. Mühürcü, "Comparative Controlling of the Lorenz Chaotic System Using the SMC and APP Methods," Mathematical Problems in Engineering, vol. 2018, pp. 1-9, 2018, doi: 10.1155/2018/9612749.

[36] V.-T. Pham, S. T. Kingni, C. Volos, S. Jafari, and T. Kapitaniak, "A simple three-dimensional fractional-order chaotic system without equilibrium: Dynamics, circuitry implementation, chaos control and synchronization," $A E U$ international Journal of Electronics and Communications, vol. 78, pp. 220-227, 2017.

[37] L. Zhang and Y. Yan, "Discrete active model predictive control of continuous unified chaotic system," in 2019 Chinese Control And Decision Conference (CCDC), 2019: IEEE, pp. 3390-3394.

[38] K.-S. Park, J.-M. Joo, J.-B. Park, Y.-H. Choi, and T.-S. Yoon, "Control of discrete-time chaotic systems using generalized predictive control," in Proceedings of 1997 IEEE International Symposium on Circuits and Systems. Circuits and Systems in the Information Age ISCAS'97, 1997, vol. 2: IEEE, pp. 789-792.

[39] S. M. Tabatabaei, S. Kamali, M. R. Jahed-Motlagh, and M. B. Yazdi, "Practical Explicit Model Predictive Control for a Class of Noise-embedded Chaotic Hybrid Systems," International Journal of Control, Automation and Systems, vol. 17, no. 4, pp. 857-866, 2019. 